\title{
Remodelamento da artéria torácica interna direita: novo método de análise pela área coronariana revascularizada
}

Remodeling of the right internal thoracic artery: new method of analysis using the coronary area

Bruno da Costa ROCHA ${ }^{1}$, Luiz Boro PUIG², Eulógio Emílio MARTINEZ FILHO ${ }^{3}$, Sérgio Almeida de

RBCCV 44205-809

\section{Resumo}

Objetivo: Avaliar os fatores angiográficos no remodelamento da artéria torácica interna (ATI) direita por novo método quantitativo com a área coronariana revascularizada.

Método: No período entre janeiro de 1992 e 2002, 452 pacientes foram submetidos à revascularização do miocárdio (RM) com ATI bilateral. Desta amostra, 32 pacientes com ponte de ATI direita "in situ" pelo seio transverso foram reestudados por meio de cineangiocoronariografia (CATE). Os filmes foram analisados na plataforma CASS II ${ }^{\circledR}$. Quanto aos critérios angiográficos, foram medidos os diâmetros proximal e distal das ATIs, área coronariana da artéria revascularizada, pontuação pela escala de fluxo de TIMI, diâmetro de estenose proximal, dominância de fluxo e presença de ramos acessórios patentes.

Resultados: O período médio de acompanhamento foi de 42 meses, variando de 6 a 204 meses. As medidas da ATID proximal foram de $2,639 \mathrm{~mm} \pm 0,09$ e distal de $2,159 \mathrm{~mm} \pm$ $0,1(p<0,001)$. A variável área coronariana correspondente à artéria marginal apresentou um coeficiente de $\mathrm{B}$ de $\mathbf{0 , 4 2 4}$ $(\mathbf{p}<0,001)$ e o diâmetro de estenose da marginal um coeficiente de $\beta$ de $0,55(p<0,0001)$ ajustados por peso, altura, intervalo cirurgia/CATE, escala de fluxo de TIMI "flow", classificação de angina e Hipertensão Arterial Sistêmica (HAS), com R ${ }^{2}$ ajustado de $0,696(p<0,0001)$.

Conclusão: Na amostra estudada, por critérios angiográficos quantitativos, a área coronariana revascularizada e o diâmetro de estenose proximal apresentaram-se como fatores independentes no remodelamento da ATID, no pós-operatório de RM.

Descritores: Anastomose mamário-coronária. Cateterismo cardíaco. Processamento de imagem assistida por computador.

Abstract

1 - Especialista em Cirurgia Cardiovascular-SBCCV. Cirurgião Cardiovascular.

2 - Professor Associado da FMUSP. Cirurgião Cardiovascular da Divisão de Coronariopatias Cirúrgicas.

3 - Professor Titular. Chefe do Serviço de Hemodinâmica e Cardiologia Intervencionista.

4 - Professor Titular FMUSP. Diretor Científico InCor FMUSP.

Trabalho realizado no InCor HC-FMUSP.

Endereço para correspondência:

Bruno da Costa Rocha. Rua Aristóteles da Costa Leal, 140, casa 118b.

Salvador, BA. CEP: 41650-030.

Artigo recebido em dezembro de 2005 Artigo aprovado em maio de 2006 
Objective: To analyze angiographic factors related to remodeling of the right internal thoracic artery (RITA) using a new quantitative technique of the target coronary grafting area.

Method: In the period from January 1992 to 2002, 452 consecutive patients were submitted to coronary artery bypass grafting (CABG) with RITA "in situ" through the transverse sinus. In this sample, 32 patients were submitted to at least one postoperative coronariography study after receiving RITA grafts. The studies were analyzed by the CASS II ${ }^{\circledR}$ software. The angiographic criteria assessed were the proximal and distal diameters of the RITA, coronary area irrigated by the target vessel, TIMI flow score, quantitative coronary angiography(QCA), dominant flow grade and the existence of patent lateral branches of the RITA.

Results: The mean follow-up period was 42 months, ranging from 6 to 204 months. The mean proximal diameter of the

\section{INTRODUÇÃO}

Desde de 1984, Puig et al. [1,2] propuseram a utilização da artéria torácica interna (ATI) direita "in situ", passando pelo seio transverso para a revascularização dos ramos da artéria circunflexa. Outras casuísticas [3-7] apresentaram alternativas técnicas e, subseqüentemente, vieram as análises de patência e a demonstração de readaptação morfológica e funcional dos enxertos, denominado de remodelamento arterial.

Apesar da evidência de variação na patência entre a ATI esquerda e direita [3-5], os fatores causais mais descritos como preditores de oclusão foram: presença de fluxo nativo pelo leito coronariano, qualidade do leito coronariano distal, presença de ramos acessórios calibrosos levando a roubo de fluxo [8-13], características demográficas e expositivas (por exemplo, diabetes mellitus, dislipidemia, tabagismo) $[12,13]$, progressão da doença aterosclerótica, além de problemas de técnica cirúrgica [14-17].

A princípio, como descrito por Gibbons et al. [18], o remodelamento vascular é um fenômeno presente nas artérias sistêmicas e coronárias, responsável pelo aumento proporcional de calibre quando em vigência de aterosclerose.
RITA was of $2.639 \mathrm{~mm} \pm 0.09$ and the distal diameter was 2.159 $\mathrm{mm} \pm 0.1(\mathrm{p}<0.001)$. The coronary irrigated by the target marginal branch presented a $\beta$ coefficient of $0.424(p=0.001)$ and diameter of the stenosis (QCA) of the marginal branch gave a $B$ coefficient of $0.55(p=0.001)$ adjusted by weight, height, time surgery/study, TIMI flow score, angina functional class and systemic hypertension. Multiple regression modeling demonstrated an adjusted $\mathrm{R} 2$ of $0.696(\mathrm{p}<0.0001)$.

Conclusion: This study, using angiographic evaluation, demonstrated that the coronary area and proximal diameter (QCA) of the marginal branch stenosis were independent factors in RITA remodeling in the postoperative of CABG.

Descriptors: Internal mammary-coronary artery anastomosis. Heart catheterization. Image processing, computer-assisted.

Este um processo fisiopatológico de readaptação de fluxo. A resposta vascular à mudança de fluxo, ao exemplo de um enxerto, modifica as forças de cisalhamento. Relaciona, portanto, o comportamento do enxerto numa situação de mudança de fluxo, modulando o vaso a aumentar ou diminuir seu diâmetro cronicamente para adaptar-se à nova condição. Barner et al. [19] determinaram uma correlação positiva entre as forças de cisalhamento sobre a parede do vaso, sendo este um dos principais estímulos para o remodelamento arterial positivo nos enxertos de artéria radial e ATI no pós-operatório de revascularização do miocárdio (RM).

A mensuração e a quantificação do remodelamento arterial fazem-se pela variação nos diâmetros luminais por angiografia e pela medida do fluxo sanguíneo pelo enxerto. Assim, o vaso submetido aguda ou cronicamente a uma modificação no seu padrão de fluxo consegue suprir adequadamente as altas demandas de oxigênio do leito receptor (por exemplo, miocárdio revascularizado) por meio do remodelamento de sua citoarquitetura, visando ao aumento da vazão de sangue [20-22].

A disponibilidade de métodos quantitativos de avaliação angiográfica pode definir, com maior precisão, as repercussões fisiológicas globais e regionais entre a interface enxerto e miocárdio revascularizado. Parâmetros angiográficos quantitativos, como escala de fluxo "TIMI" [23,24], 
percentual de diâmetro de estenose (QCA)[25] e dominância de fluxo, são ferrramentas de análise no remodelamento da ATID.

Com a utilização destes critérios angiográficos previamente validados, e com o novo método de medida da área coronariana revascularizada, o presente estudo tem o objetivo de avaliar a influência de cada fator angiográfico e clínico no remodelamento da ATID.

\section{MÉTODO}

\section{População envolvida}

O estudo tem desenho retrospectivo baseado em exames de cineangiocoronariografia feitos no pós-operatório de revascularização do miocárdio, por indicação clínica e independentemente deste protocolo de pesquisa. A Comissão Científica e de Ética do InCor-HCFMUSP e a Comissão de Ética para Análise de Projeto de Pesquisa (CAPPesq) aprovou o projeto, classificando-o como "sem risco", sendo protocolado com $N^{\circ} 574 / 03$, sob a Resolução do Conselho Nacional de Saúde 196, na data de 13 agosto de 2003. Em estudo-piloto preliminar, foi determinado cálculo de amostra para um erro de $\beta$ de 0,2 e $\alpha$ de $0,05, n=30$.

\section{Critérios de seleção}

Critérios de inclusão

1. Presença da ATD patente;

2. Ausência de lesão $>20 \%$ na ATID ou na anastomose;

3. Qualidade técnica suficiente para análise do exame ou para "digitalização" do filme;

4. Na presença de mais de um exame por paciente em períodos diferentes, o que completar de forma suficiente a adequação técnica e com maior intervalo de tempo cirurgia/ reestudo será incluído.

\section{Critérios de exclusão}

1. Inadequação técnica para análise "a posteriori” do filme na plataforma CASS II® ou Osiris v.11®;

2. Inadequação de projeções em plano ortogonal suficiente para todas as medidas angiográficas propostas.

\section{Padronização técnica}

A análise dos exames de cineangiocoronariografia foi realizada por dois observadores independentes. Após conformidade dos observadores quanto à possibilidade técnica de manipulação das imagens, os filmes precedentes a 1995 foram "digitalizados" em formato "DICOM". Posteriormente, foram analisados pelos "software" CASS II ${ }^{\circledR}$ e Osiris v.11®. Quanto aos critérios angiográficos, foram medidos os diâmetros proximal e distal das ATIs, área coronariana da artéria revascularizada, pontuação pela escala de TIMI "flow", graduação do diâmetro de estenose pelo leito nativo, determinação da dominância de fluxo e determinação da presença de ramos acessórios procedente da ATI.

Foi utilizado um microcomputador Macintoch com "interface" com a plataforma CASS II ${ }^{\circledR}$ para exames diretamente em sistema de rede interna, ou por meio da "digitalização" de imagens para formato "TIF" e "DICOM" procedente de filmes em VHS, S-VHS ou $35 \mathrm{~mm}$.

A calibração das imagens foi realizada por meio da medida da ponta do cateter sem contraste. A resultante desta calibração consiste na relação entre o número de "pixels" e a medida previamente conhecida do cateter em milímetros; por exemplo, cateter tipo Judkins 7F, a ponta possui $2,33 \mathrm{~mm}$, o que hipoteticamente corresponderia a 12 "pixels", naquelas condições de penetração e profundidade de imagem. Uma medida de 24 "pixels" corresponderia,

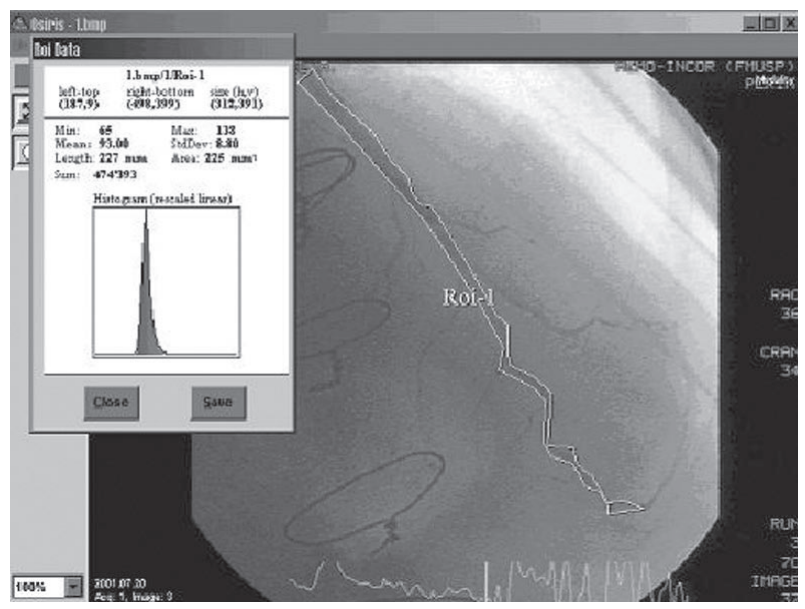

Fig. 1 - Delimitação da área coronariana do ramo interventricular anterior, anexo histograma sobre a área de interesse

portanto, a 4,66 mm. As medidas foram realizadas no plano ortogonal mais adequado. A delimitação da área de interesse foi feita pela demarcação automática. Quanto à correção das distorções, sobreposições de imagens foram feitas manualmente.

\section{Critérios angiográficos}

Diâmetro proximal e distal: Foi delimitada uma área de interesse de extensão entre 15 e $20 \mathrm{~mm}$, a $20 \mathrm{~mm}$ da origem da ATI na artéria subclávia ou ramo e a $20 \mathrm{~mm}$ proximal da anastomose entre a ATI e a artéria coronária revascularizada. Foi realizada análise do gráfico de área, com as medidas de área circular, área de referência e diâmetro de referência. $\mathrm{O}$ diâmetro de referência é a resultante da média de diâmetro da área de interesse e corresponde ao diâmetro proximal.

Área coronária revascularizada: medida a partir da anastomose entre a ATI e a artéria coronária revascularizada, em sentido distal foi demarcada toda a superfície coronariana e principais ramos. Na condição de fluxo retrógradq $q_{\mathbf{i}}^{\grave{i}}$ 
anastomose, também foi medida a superfície coronariana nas mesmas condições, e razão entre ambas considerada. A medida da área coronariana é expressa em milímetros quadrados (Figura 1).

TIMI "flow": Conforme o critério de TIMI, numa escala de 0 a 3 , foi pontuado o fluxo através do leito coronariano distal e considerado a maior pontuação, seja através da injeção pelo leito nativo ou pela ATI (TIMI 0sem fluxo, TIMI 1- fluxo sem perfusão, TIMI 2- fluxo lento, TIMI 3- normofluxo).

Dominância de fluxo: A dominância de fluxo pelo leito coronariano revascularizado pode ser balanceado, dominante pelo leito nativo ou dominante pela ATI (2- enxerto de ATI, 1- balanceado, 0- leito coronariano nativo).

Diâmetro de estenose do leito coronariano revascularizado: Pelo método de QCA foi delimitado, no leito coronariano revascularizado, o local de estenose; desta forma, o diâmetro de referência é resultante da média de diâmetro proximal e distal à placa aterosclerótica. Medido em percentual de estenose (0-100\%).

Presença de ramos acessórios da ATI: A determinação de ramos acessórios patentes, correspondentes principalmente

Tabela 1. Características demográficas da amostra

\begin{tabular}{lc}
\hline & Características da amostra \\
\hline Idade(anos) & $63,1 \pm 1,5 *$ \\
& $(42-79) * *$ \\
Peso(kg) & $73,8 \pm 7,9$ \\
& $(58-92)$ \\
Altura(m) & $1,66 \pm 0,08$ \\
& $(1,35-1,82)$ \\
Sexo masculino & $26(81,6 \%)^{* * *}$ \\
Dislipidemia & $14(43,8 \%)$ \\
DM & $6(18,8 \%)$ \\
HAS & $19(59,4 \%)$ \\
Tabagismo & $8(25 \%)$
\end{tabular}

Classificação de

angina

Estável

Instável

$12(37,5 \%)$

$14(43,8 \%)$

Assintomático

$6(18,8 \%)$

$\begin{array}{lc}\text { CF NYHA } & 9(28,1 \%) \\ \text { I } & 9(28,1 \%) \\ \text { II } & 2(6,3 \%) \\ \text { III } & 0\end{array}$

*média \pm ep; **valores mínimo e máximo; ***n (percentual relativo); DM - Diabetes Mellitus, HAS - Hipertensão Arterial Sistêmica, CF-Classe Funcional pela classificação do NYHA - New York Heart Association. aos ramos costo-lateral, pericárdio-frênico e intercostal. A medida do diâmetro acima de $1 \mathrm{~mm}$ foi considerada significativa (0-ausente, 1-presente).

\section{Análise estatística}

A análise foi realizada em duas fases. Numa primeira fase, as variáveis foram categorizadas quanto à freqüência de distribuição. O diâmetro distal da ATI foi considerado como variável dependente. As variáveis independentes paramétricas foram submetidas ao teste $\mathrm{t}$ de Student; as variáveis não-paramétricas, à correlação de Spearman, teste de Qui-quadrado e teste Mann-Whitney. Numa segunda fase, as variáveis que apresentaram um $\mathrm{p}<0,1$ foram submetidas a um modelo de regressão linear múltiplo. Foi considerado um nível de significância estatística de 0,05.

\section{RESULTADOS}

Tabela 2. Teste t-Student ou Mann-Whitney entre as médias de diâmetro

\begin{tabular}{|c|c|c|c|}
\hline \multicolumn{4}{|c|}{ Artéria Torácica Interna Direita } \\
\hline & Presente & Ausente & $\mathrm{p}$ \\
\hline Sexo feminino & $\begin{array}{c}2,055 \pm 0,152 \\
(\mathrm{n}=6)\end{array}$ & $\begin{array}{c}2,0 \pm 0,108 \\
(\mathrm{n}=26)\end{array}$ & 0,82 \\
\hline Ramos acessórios & $\begin{array}{c}1,937 \pm 0,181 \\
(\mathrm{n}=8)\end{array}$ & $\begin{array}{c}2,035 \pm 0,107 \\
(\mathrm{n}=24)\end{array}$ & 0,65 \\
\hline Dislipidemia & $\begin{array}{c}2,072 \pm 0,137 \\
(\mathrm{n}=14)\end{array}$ & $\begin{array}{c}1,962 \pm 0,124 \\
(\mathrm{n}=18)\end{array}$ & 0,55 \\
\hline Diabetes Mellitus & $\begin{array}{c}1,951 \pm 0,29 \\
(\mathrm{n}=6)\end{array}$ & $\begin{array}{c}2,024 \pm 0,094 \\
(\mathrm{n}=26)\end{array}$ & 0,76 \\
\hline \multicolumn{4}{|l|}{ Hipertensão Arterial } \\
\hline Sistêmica & $\begin{array}{c}1,886 \pm 0,122 \\
(\mathrm{n}=19)\end{array}$ & $\begin{array}{c}2,192 \pm 0,126 \\
(n=13)\end{array}$ & 0,10 \\
\hline IAM prévio & $\begin{array}{c}1,887 \pm 0,103 \\
(\mathrm{n}=15)\end{array}$ & $\begin{array}{c}2,119 \pm 0,144 \\
(\mathrm{n}=17)\end{array}$ & 0,21 \\
\hline Tabagismo & $\begin{array}{c}1,817 \pm 0,223 \\
(\mathrm{n}=8)\end{array}$ & $\begin{array}{c}2,075 \pm 0,096 \\
(\mathrm{n}=24)\end{array}$ & 0,22 \\
\hline
\end{tabular}

distal em milímetros da ATID com as variáveis independentes *média $\pm 2 E P$

Tabela 3. Correlação de Spearman entre a média de diâmetro distal das ATID e variáveis independentes

\begin{tabular}{lcc}
\hline & $\begin{array}{c}\text { Coeficiente de } \\
\text { correlação (rho) }\end{array}$ & $\begin{array}{c}\text { Significância } \\
\text { bicaudal }\end{array}$ \\
\hline Peso & $-0,35$ & 0,05 \\
Altura & $-0,16$ & 0,03 \\
Idade & $-0,06$ & 0,74 \\
Área coronariana & 0,67 & $<0,0001$ \\
Intervalo cirurgia/CATE & 0,29 & 0,1 \\
Diâmetro de estenose proximal & 0,32 & 0,08 \\
Critério de fluxo TIMI & 0,35 & 0,05 \\
Dominância de fluxo & $-0,21$ & 0,24 \\
CF-NYHA & $-0,19$ & 0,29 \\
Classificação de Angina & $-0,35$ & 0,05 \\
\hline
\end{tabular}


No período analisado foram identificados 452 pacientes submetidos à RM, com dupla ATI. Desta amostra foram estudados, por cineangiocoronariografia, 32 pacientes com ponte de ATID "in situ" pelo seio transverso. O período médio de acompanhamento foi de 42 meses, variando de 1 a 204 meses entre a cirurgia e o reestudo angiográfico.

A amostra foi constituída pelas seguintes características clínicas e demográficas (Tabela 1). O diâmetro distal do enxerto de ATID foi comparado por teste de hipótese entre as características demográficas e angiográficas (Tabela 2).

As variáveis independentes contínuas ou ordinais foram correlacionadas com o diâmetro distal da ATID (Tabela $3)$.

As variáveis: peso, altura, área coronariana, intervalo cirurgia/CATE, diâmetro de estenose proximal, TIMI, classificação de angina e HAS apresentaram-se significante estatisticamente na análise univariada (Tabelas 2 e 3 ).

No modelo de regressão linear múltiplo, foram incluídos oito variáveis independentes. A área coronariana correspondente à artéria marginal apresentou um coeficiente de $\beta$ de $0,424(\mathrm{p}<0,001)$ e o diâmetro de estenose da marginal, um coeficiente de $\beta$ de $0,55(p<0,0001)$. Este modelo apresentou $\mathrm{R}=0,846$ e $\mathrm{R}^{2}$ ajustado $=0,696 \mathrm{com}$ $\mathrm{p}<0,0001$.

\section{DISCUSSÃO}

A análise dos fatores angiográficos para avaliação do remodelamento de enxertos arteriais baseia-se "in vivo" em estudo morfométrico. Pela análise morfométrica, o presente estudo focou-se em critérios angiográficos validados [2325], fatores de influência direta sobre o enxerto de ATI [916] e mensurou quantitativamente o leito coronário distal pela medida de área.

A análise univariada, a área coronária do ramo marginal da artéria circunflexa, o tempo em meses entre a cirurgia e o reestudo por CATE e o diâmetro de estenose no ramo marginal apresentaram correlação positiva e moderada com o diâmetro da ATID. Outras variáveis foram incluídas no modelo de regressão, não obstante por serem consideradas possíveis variáveis de confusão, servindo para ajustar o modelo proposto. Notadamente, com um modelo ajustado, variáveis de menor ou nenhuma magnitude, como a presença de ramos colaterais patentes, outrora correlacionado com o remodelamento negativo [9-13] e "roubo" de fluxo, não demonstraram correlação estatisticamente significativa.

É notória a influência do leito nativo distal, medido pela área da marginal revascularizada, com o remodelamento positivo da ATID. Esta medida possuiu coeficiente de regressão positivo com moderada importância e independente de outras possíveis variáveis de confusão como altura, peso, idade e sexo. Quanto aos coeficientes de $\beta$, estes são resultantes finais de um modelo estatístico baseado em regressão linear múltiplo. Portanto, pode-se interpretá-lo como fatores independentes de correlação com o desfecho analisado. Para tanto, neste estudo, o diâmetro distal da ATID, considerado como variável dependente, submeteu-se a influência da área coronariana revascularizada em 0,42 $(\mathrm{p}<0,001)$ e do diâmetro de estenose da marginal com 0,55 $(p<0,0001)$. A aplicação destes coeficientes demonstrou um valor preditivo sobre diâmetro da ATID, ou seja, estes podem isoladamente determinar a capacidade e magnitude de alteração dos diâmetros de ATID.

Este modelo apresentou $\mathrm{R}^{2}$ ajustado $=0,696 \mathrm{com}$ $\mathrm{p}<0,0001$. Isto denota a probabilidade de $69,6 \%$ de capacidade deste modelo estatístico, pelos coeficientes finais, de predizer a medida de diâmetro do enxerto de ATID, independentemente às influências de tantas variáveis inseridas inicialmente no modelo, e que obtiveram significância estatística apenas na análise univariada.

Outros autores [19-21] também observaram correlação positiva entre o diâmetro de estenose e do enxerto. A presença de estenose no leito nativo, de maior ou menor magnitude, influencia na dominância de fluxo pelo leito distal (fluxo

\section{REFERÊNCIAS}

1. Puig LB, Franca-Neto L, Rati M, Ramires JA, Luz PL, Pileggi $\mathrm{F}$ et al. A technique of anastomosis of the right internal mammary artery to the circumflex artery and its branches. Ann Thorac Surg. 1984;38(5):533-4.

2. Gerola LR, Puig LB, Moreira LF, Cividanes GV, Gemha GP, Souto RCM et al. Right internal thoracic artery through the transverse sinus in myocardial revascularization. Ann Thorac Surg. 1996;61(6):1708-13.

3. Puig LB, Ciongolli W, Cividanes GVL, Teofilo Junior S, Dontof AC, Fiorelli AI et al. Artéria epigástrica inferior como enxerto livre. Uma nova alternativa na revascularização direta do miocárdio. Arq Bras Cardiol. 1988;50(4):259-61.

4. Lytle BW, Blackstone EH, Loop FD, Houghtaling PL, Arnold JH, Akhrass R et al. Two internal thoracic artery grafts are better than one. J Thorac Cardiovasc Surg. 1999;117(5):855-72. 
5. Ura M, Sakata R, Nakayama Y, Arai Y, Saito T. Long-term patency rate of right internal thoracic artery bypass via the transverse sinus. Circulation. 1998;98(19):2043-8.

6. Taggart DP. Respiratory dysfunction after cardiac surgery: effects of avoiding cardiopulmonary bypass and the use of bilateral internal mammary arteries. Eur J Cardiothorac Surg. 2000;18(1):31-7.

7. Schmidt SE, Jones JW, Thornby JI, Miller III CC, Beall AC Jr. Improved survival with multiple left-sided bilateral internal thoracic artery grafts. Ann Thorac Surg. 1997;64(1):9-15.

8. Galbut DL, Traad EA, Dorman MJ, DeWitt PL, Larsen PB, Kurlansky PA et al. Seventeen-year experience with bilateral internal mammary artery grafts. Ann Thorac Surg. 1990;49(2):195-201.

9. Singh RN, Sosa JA. Internal mammary artery-coronary artery anastomosis: influence of the side branches on surgical result. J Thorac Cardiovasc Surg. 1981;82(6): 909-14.

10. Hartz RS, Heuser RR. Embolization of IMA side branch for post-CABG ischemia. Ann Thorac Surg. 1997;63(6):1765-6.

11. Kern MJ, Bach RG, Donohue TJ, Caracciolo EA, Wolford T, Aguirre FV. Interventional physiology. Part XIII: Role of large pectoralis branch artery in flow through a patent left internal mammary artery conduit. Cathet Cardiovasc Diagn. 1995;34(3):240-4.

12. Luise R, Teodori G, Di Giammarco G, D’Annunzio E, Paloscia L, Barsotti A et al. Persistence of mammary artery branches and blood supply to the left anterior descending artery. Ann Thorac Surg. 1997;63(6):1759-64.

13. Kern MJ. Mammary side branch steal: is this a real or even clinically important phenomenon. Ann Thorac Surg. 1998;66(6):1873-5.

14. Seki T, Kitamura S, Kawachi K, Morita R, Kawata T, Mizuguchi $\mathrm{K}$ et al. A quantitative study of postoperative luminal narrowing of the internal thoracic artery graft in coronary artery bypass surgery. J Thorac Cardiovasc Surg. 1992;104(6):1532-8.

15. Hashimoto H, Isshiki T, Ikari Y, Hara K, Saeki F, Tamura T et al. Effects of competitive blood flow on arterial graft patency and diameter: medium-term postoperative follow-up. J Thorac Cardiovasc Surg. 1996;111(2):399-407.

16. Huddleston CB, Stoney WS, Alford WC Jr, Burrus GR, Glassford DM Jr, Lea JW et al. Internal mammary artery grafts: technical factors influencing patency. Ann Thorac Surg. 1986;42(5):543-9.

17. Dion R, Glineur D, Derouck D, Verhelst R, Noirhomme $\mathrm{P}$, Khoury $\mathrm{G}$ et al. Long-term clinical and angiographic follow-up of sequential internal thoracic artery grafting. Eur J Cardiothorac Surg. 2000;17(4):407-14.

18. Gibbons GH, Dzau VJ. The emerging concept of vascular remodeling. N Eng J Med. 1994;330(20):1431-8.

19. Barner HB. Remodeling of arterial conduits in coronary grafting. Ann Thorac Surg. 2002;73(4):1341-5.

20. Glagov S, Weisenberg E, Zarins CK, Stankunavicius R, Kolettis GJ. Compensatory enlargement of human atherosclerotic coronary arteries. N Engl J Med. 1987;316(22):1371-5.

21. Kouchi Y, Onuki Y, Wu MH, Shi Q, Sauvage LR. Effect of altered blood flow on caliber and morphology of the internal thoracic artery in the dog. J Thorac Cardiovasc Surg. 1997;113(1):114-20.

22. Baumbach GL, Heistad DD. Remodeling of cerebral arterioles in chronic hypertension. Hypertension. 1989;13(6 pt 2):968-72.

23. LincoffAM, Topol EJ, Califf RM, Sigmon KN, Lee KL, Ohman EM et al. Significance of a coronary artery with trombolysis in myocardial infarction grade 2 flow "patency"(outcome in the thrombolysis and angioplasty in myocardial infarction trials. Am J Cardiol. 1995;75(14):871-6.

24. Gibson CM, Murphy SA, Rizzo MJ, Ryan KA, Marble SJ, McCabe $\mathrm{CH}$ et al. Relationship between TIMI frame count and clinical outcomes after thrombolytic administration. Circulation. 1999;99(15):1945-50.

25. Brown BG, Bolson E, Frimer M, Dodge HT. Quantitative coronary arteriography: estimation of dimensions, hemodynamic resistance and atheroma mass of coronay artery lesions using the arteriogram and digital computation. Circulation. 1977;55(2):329-37. 